体。鋅濛脫石的出現和分布規律的研究，能为探寻

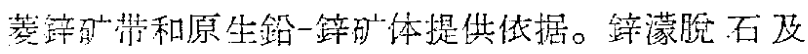
其有关共生矿物組合、綘构构造的研究能为第化矿 石的选时、治燎、綜合利用提供有利的資料。

志謝：本交蒙何作霜教授审閶，并提供意見，特此致 䶆。

[1] Ross C. S., The American Meneralogist, 31, No. 9-10, P. 411-424 (1946).

[2] Faust R. T., The American Minerulogist, 36, No. $11-12$, p. $795-822$ (1951).
[3] Гинзбург И. И., Витовская И. В., В сб.: Кора выветривания, 2 (1956).

[4] Семенов Е. И., Институт минер. геохимии и кристаллохимии редких элементов. АН СССР, Труды, 2 Стр. 124-137 (1959).

[5] Семенов Е. И., В сб.: Кора выветривания, 2, (1956).

[6] Taylor H. F. W., Amer. Mineralogist. 47, No. $7-8$, p. 932-944 (1962).

[.7] Roy D. M., Econ. Geol. 51, p. 432-443 (1956).

[8] Takahashi Tako., Econ. Geol. 55, p. 10831115 (1960).

[9] Чухров Ф. В., Қоллоиды в земной коре, ИздBO, АH CCCP, 1955.

\title{
$\mathrm{NiO}-\mathrm{Li}_{2} \mathrm{O}$ 系催化剂上氧的不同吸附态*
}

\section{王弘立黄桂惕 楼偑佩}

在氯化物推化剂上，氧的不同的吸附态，筸为 許多学者所假設与討諭 ${ }^{[1-4]}$ 。所有这些假設和討諭 一直缺泛实驗的竣証。最近，Казанский 等[5] 用順 磁共振法研究了在 $\mathrm{TiO}_{2}$ 上吸附氬的不同状态, 得 到了两种信号。他們䚿为这是由 $\mathrm{O}_{2}^{-}$及 $\mathrm{O}^{-}$所引起 的, 从而提供了在 $\mathrm{TiO}_{2}$ 催化济表面上存在着两种 带不同負电荷的直按实驗証据。另外，Maxim 及 Braun ${ }^{[6]}$ 在研究楅射对 $\mathrm{NiO}$ 上的化学吸附平衡的影 晌时，用不同的分析方法得到了表面氧有不同的含 量, 从而䚿为 $\mathrm{NiO}$ 上具有两种不同的化学吸附氧。 一种是弱化学吸附, 中性的; 另一种为强化学吸附, 带負电。本文应用四附过程中催化剂的逸出功和电 阻的变化, 考察了在 $\mathrm{NiO}-\mathrm{Li}_{2} \mathrm{O}$ 系催化剂上氧是否 以不同吸附态存在的問題。

共制备了近种 $\mathrm{NiO}-\mathrm{Li}_{2} \mathrm{O}$ 催化剂, 其中 $\mathrm{Li}_{2} \mathrm{O}$ 的 加入量分別相应于 $\mathrm{Li} / \mathrm{Ni}$ 原子百分比为 $0 、 0.08$ 、 0.8、4 及 $8 \%$ 。 NiO 由碳酸䤼制成，将碳酸䤼在 $600^{\circ} \mathrm{C}$ 分解三小时, 冷却至室溫, 粉碎后在 3000 大 气压下压制成片状，最后在空气中 $\left(1000^{\circ} \mathrm{C}\right)$ 活化 8 小时。 $\mathrm{NiO}-\mathrm{Li}_{2} \mathrm{O}$ 是用 $\mathrm{NiCO}_{3}$ 加入到 $\mathrm{Li}_{2} \mathrm{CO}_{3}$ 的 醋酸溶液中，㨨汼均匀后，再进行同样条件的分解 和活化制成。这五种催化剂的 BET 浸面积, 随着 $\mathrm{L}_{2} \mathrm{O}$ 加入量的坦加而減小，分別为 $0.33 、 0.33$ 、

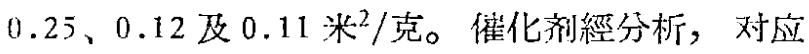
于四个含鋰催化剂, 鋰的实际含量分別为 0.04 、 $0.40 、 1.9$ 及 3.8 原子\% $\mathrm{Li}_{\text {。 }}$

催化剂經 $400^{\circ}$ 抽空 4 小时, 作为吸附前予处 理, 实驗温度为 $260^{\circ}$, 氧的初压为 $\sim 0.1$ 毫米永桂, 用 MeLeod 真空計測量压力的变化，以計算吸附量。 在測量吸附量的同时，用振动电容法測量催化剂的 逸出功, 扗在吸附进行时用交流电桥測量电阻的变 化。

* 本文曾在全国第二次催化磁究工作报告会上宣讀。 
在五种催化剂样品上氧的吸附动力学等溫綫見 图1。鋰加入量高都 $(\mathrm{NiO}+4$ 原子\% Li 及 $\mathrm{NiO}$ +8 原子 $\% \mathrm{Li})$ 的初始吸附速度远較鋰加入量低者 $(\mathrm{NiO} 、 \mathrm{NiO}+0.08$ 原子 $\% \mathrm{Li}$ 支 $\mathrm{NiO}+0.8$ 原子 $\%$ $\mathrm{Li}$ ) 为大, 而后者, $\mathrm{NiO}>\mathrm{NiO}+0.08$ 原子 $\% \mathrm{Li}>$. $\mathrm{NiO}+0.8$ 原子 $\% \mathrm{Li}$ 。

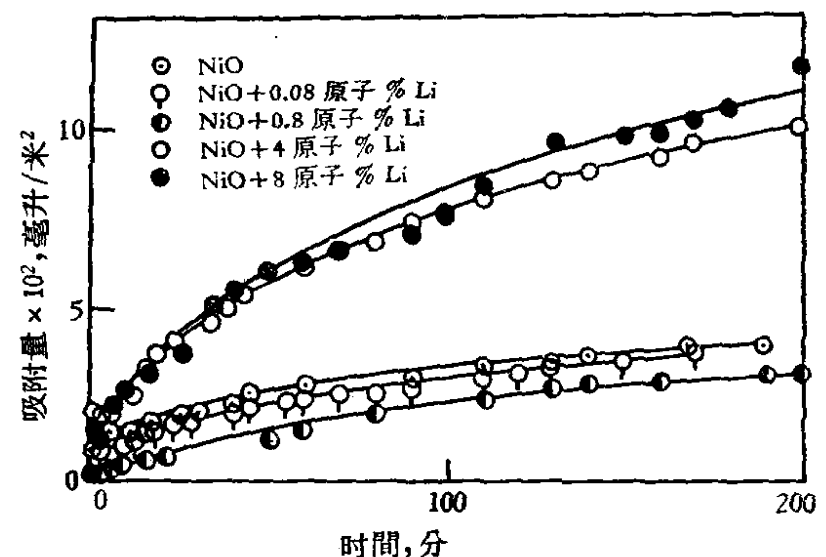

图 1 氧的吸附动力学等溫綫 $T=260^{\circ} ; P_{0}=0.1$ 毫米乘柱

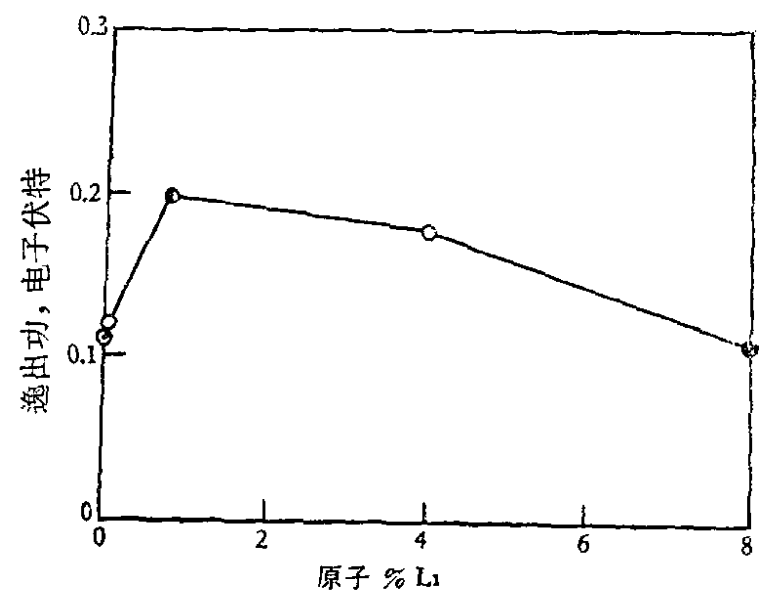

图 2 在虽空下的催化剂逸出功 $T=260^{\circ}$

$\mathrm{NiO}-\mathrm{Li}_{2} \mathrm{O}$ 系催化剂在 $260^{\circ}$ 时真空中相对于鎳 的逸出功数值如图 2 所示。在 $\mathrm{NiO}+0.8$ 原子 \% $\% \mathrm{i}$ 处有一高峯, 根据 Kуцева 及 Keh̆ep ${ }^{[7]}$ 引用 Germain 所提出的見解，对于受主气体的吸附，如氧，吸附 热随催化剂的逸出功增加而減小，再根据 Brönsted 的經驗关系式，吸附热愈大則吸附活化能愈小，汃 臫一般說来吸附嗃度愈大。因此，从催化剂的逸出 功数据, 可以予期吸附速度在 $\mathrm{NiO}+0.8$ 原子\% $\%$ Li 处有一最低徝，如图 1 所示。
从图 1 还可以看到, 在所有催化剂上, 嵫的吸 附量随着时間不断增加, 吸附速度則随着时間而減 小，在約 200 分鈡时方才接近本衡。此的睖附量不 再增加或增加很少。如将此吸附量作为平衡吸附 量，則当鋰加入量較低时，其值随着鋰加入量的管 加而減小，郎 $\mathrm{NiO}>\mathrm{NiO}+0.08$ 原子 $\% \mathrm{Li}>\mathrm{NiO}$ +0.8 原子 \% Li; 高鋰加入量催化剂上氧的平衡吸 附量約为低鋰加入量者的三倍,而 $\mathrm{NiO}+8$ 原子 $\% \mathrm{Li}$ 的又高于 $\mathrm{NiO}+4$ 原子 $\% \mathrm{Li}$ 的。

逸出功在吸附初期約 20 分徐的改变很大。莪 的吸附使催化剂的逻出功增加，20 分鈡后趋近平 稳，或者改变很少，或者根本不丽改变，見图 3 。 电阻在吸附开始时亦改变很快。氧的吸附使催化剂 的电阻下降，亦在約 20 分錦时达到一稳定谊，見 图 4。

由于 $\mathrm{NiO}$ 是 $\mathrm{p}$ 型牛导体， $\mathrm{NiO}-\mathrm{Li}_{2} \mathrm{O}$ 亦是 $\mathrm{p}$ 型 牛导体, 逸出功因氧的吸附而增加以及电阻因藏的 吸附而降低,都表示氧在吸附时自催化剂接受忠广， 从而形成带負电的粒于。逸出功和电阻同时在約 20 分鈡后达到一稳定值, 而四附量却仍然不䁋增加, 在約 200 分鈡时方才接近华衡。这說明吸附的氧以 两种不同的状态存在，一种是带負电的，一种是不 带电的，与 Maxim 及 Braun ${ }^{[6]}$ 的結諭一致。

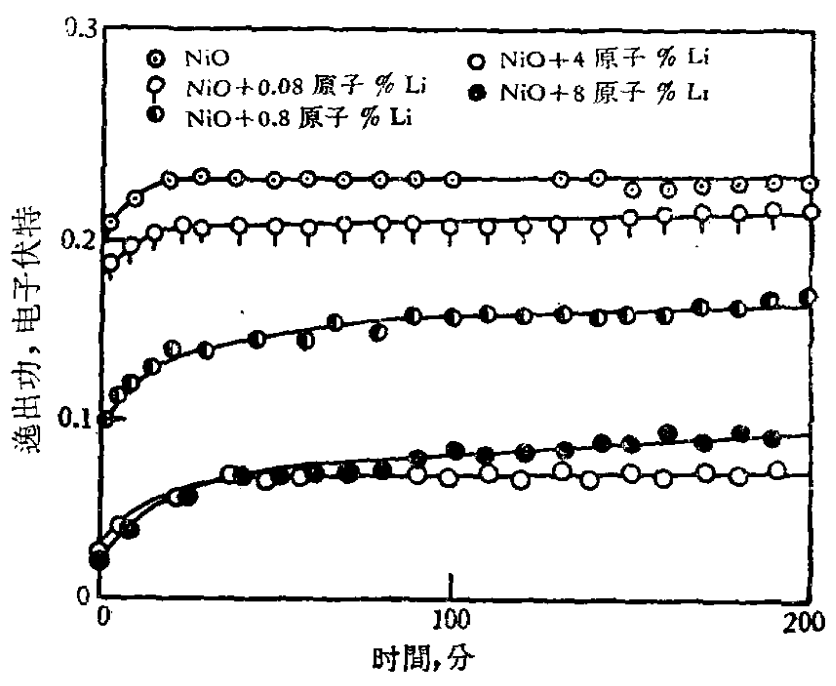

图 3 在氧吸附进行时僬化剂透出功的变化 $T=260^{\circ} ; P_{0}=0.1$ 毫米我杜

如果挦定氧的带电吸附态为 $\mathrm{O}^{-}$, 不带电的吸附 态为 O 或如 Grimeley 及 Trapnel1[8] 在討詥金属的 


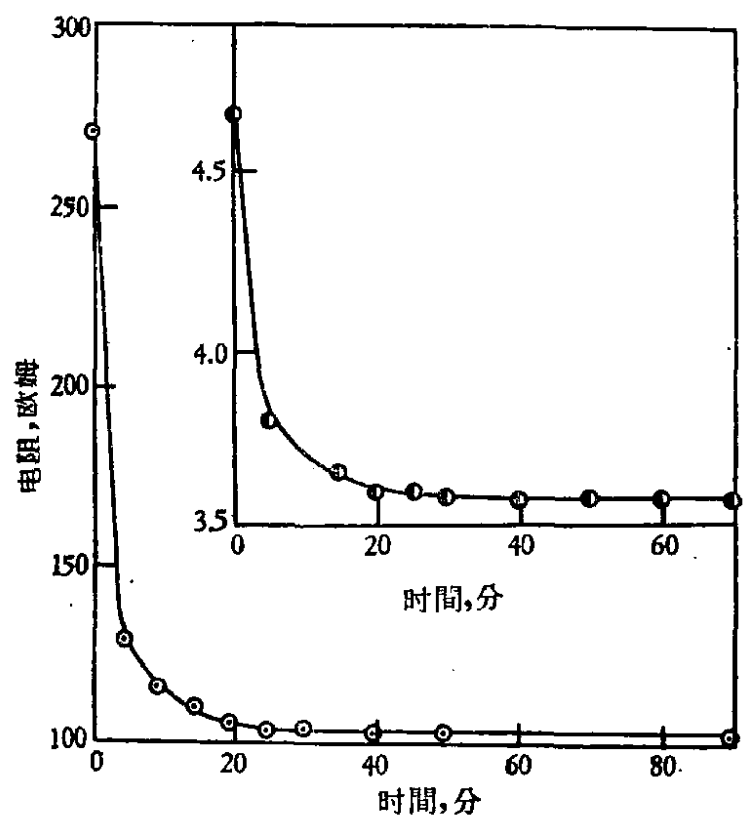

图 4 在氧吸附进行时催化剂电阻的变化 $T=260^{\circ} P_{0}=0.1$ 撉米采桂 ○ $\mathrm{NiO} \bigcirc \mathrm{NiO}+0.8$ 原子\% Li

氧化机理时所假設的所謂中性对,垖伴有空穴的 $\mathrm{O}^{-}$， 則可以根据 Krusemyer 及 Thomas ${ }^{[9]}$ 对牛导体表面 上气体吸附平衡的一般处理, 从由于氧的吸附而引 起的逸出功变化 $\Delta \phi$, 可近似地按下式計算单位表 面上带急电吸附粒子的数目 $\left[\mathrm{O}^{-}\right]$:

$$
\begin{aligned}
{\left[\mathrm{O}^{-}\right] } & =\left(2 k T \epsilon \epsilon_{0} / e^{2}\right)^{1 / 2} P_{\infty}^{1 / 2} \\
& \times[\exp (e \Delta \phi / k T)-1-e \Delta \phi / k T]^{1 / 2}
\end{aligned}
$$

式中 $k$ 为玻尔茹曼常数； $\epsilon$ 为介电常数，計算时設 $\mathrm{NiO}-\mathrm{Li}_{2} \mathrm{O}$ 系催化剂的介电常数为 $10 ; \epsilon_{0}$ 为电容率; $e$ 为电子电荷; $P_{\infty}$ 为催化剂体相队的空穴浓度, 計 算时假定杂盾全部电窝, 則 $P_{\infty}=5.5 \times 10^{22} \times \mathrm{Li}$ 原子 $\%+\left(P_{\infty}\right)_{N i O}, \mathrm{NiO}$ 的数值則从电导活化能估 算 $[10]$ 。所得的結果列于表 1 。再将 200 分鈡时的吸 附量作为平衡吸附量, 就可以計算达到吸附平衡时 带真电粒子的分数, 邲 $\left[\mathrm{O}^{-}\right] /\left[\mathrm{O}^{-}\right]+[$中性氧 $]$, 計 算値亦列于表 1 。

氧吸附动力学等 溫 綫与 Куцева 及 Кейер ${ }^{[7}$ 所报导的恰恰相反。她們的数据是在 $T=353^{\circ}$,
表 1 NiO- $\mathrm{Li}_{2} \mathrm{O}$ 系催化剂, 在 0.1 毫米禾桂 压力下达到吸附平衡时, 带資电吸附氧

\begin{tabular}{|c|c|c|}
\hline 催 化 剂 & {$\left[\mathrm{O}^{-}\right] /$厘米 $^{2}$} & $\begin{array}{c}{\left[\mathrm{O}^{-}\right] /\left[\mathrm{O}^{-}\right]+} \\
{[\text {中性氧] }}\end{array}$ \\
\hline $\mathrm{NiO}$ & $6.0 \times 10^{13}$ & $-30 \%$ \\
\hline $\begin{array}{c}\mathrm{NiO}+0.08 \\
\text { 原子\% Li }\end{array}$ & $7.3 \times 10^{13}$ & $\sim 40 \%$ \\
\hline $\begin{array}{l}\mathrm{NiO}+0.8 \\
\quad \text { 原子 } \% \mathrm{Li}\end{array}$ & $7 \cdot 2 \times 10^{1.3}$ & $\sim 45 \%$ \\
\hline $\begin{array}{l}\mathrm{NiO}+4 \\
\quad \text { 原子\% Li }\end{array}$ & $3.9 \times 10^{13}$ & $\sim 10 \%$ \\
\hline $\begin{array}{l}\mathrm{NiO}+8 \\
\quad \text { 原子\% Li }\end{array}$ & $7.1 \times 10^{13}$ & $\sim 10 \%$ \\
\hline
\end{tabular}
粒子的表面浓度及分数

$P=0.08$ 毫米永柱的条件下取得的。初始时的吸 附速度在鋰加入量較低时, 随着鋰加入量的買加而 增加，吸附活化能相应減小; 而当鋰加入量較高时 $(\mathrm{NiO}+8$ 原于\% $\mathrm{Li})$, 吸附速度反而最低,吸附活化 能則最高。这些不同的結果是由于样品的不同, 或 由于其它原因所造成，目前份难解释。

志謝：催化剂中鋰含量的分析是在万邦和同志的指导 下由张玉林、王风英和何桂森等同志測定的，謰此致謝。

[1] Волькенштейн Ф. Ф., Электронная теория катализа на полупроводниках, Москва, 1960.

[2] Parravano G., Boudart M., Adavances in Catalysis, 7, 50 (1955).

[3] Марголис П. Я., Усп. хим., 28, 611 (1959).

[4] Barry T. I. and Stone F. S., Proc. Royal Soc., 255, 124 (1960).

[5] Казанский В. Б., Никитина О. В., Париснй, Г. Б., ДАН СССР, 151, № 2, 369 (1963).

[6] Maxim I, and Braun T., J. Phys. Chem. Solids, 24, 537 (1963).

[7] Куцева Л, Н., Кейер, Н. П., Прблемь кинетики и катализа, 10, 82 (1960).

[8] Grimley T. B. and Trapnell B. M. W., Proc. Royal Soc., A234, 405 (1956).

[9] Krusemeyer H. J. and Thomas D. G., J. Phys, Chem. Solids, 4, 78 (1958).

[10] Van Houton S., J. Phys. Chem. Solids, 17, T $(1960)$. 